Ciência Florestal, Santa Maria, v. 22, n. 4, p. 865-875, out.-dez., 2012

ISSN 0103-9954

\title{
FUNDAMENTOS E ESTADO DA ARTE DA ESPECTROSCOPIA NO INFRAVERMELHO PRÓXIMO NO SETOR DE BASE FLORESTAL
}

\section{BACKGROUND AND STATE OF THE ART OF NEAR INFRARED SPECTROSCOPY IN THE FOREST SECTOR BASE}

\author{
Graciela Inês Bolzon de Muñiz ${ }^{1}$ Washington Luiz Esteves Magalhães ${ }^{2}$ \\ Mayara Elita Carneiro ${ }^{3}$ Lívia Cássia Viana ${ }^{4}$
}

\begin{abstract}
RESUMO
O conhecimento das propriedades da madeira é de fundamental importância para indicação dos potenciais de utilização final deste material. Na busca por novas alternativas para caracterização rápida, simples e confiável, destacam-se as avaliações não destrutivas da madeira. A espectroscopia no infravermelho próximo (NIRS) vem sendo utilizada como método não destrutivo que permite obter informações qualitativas e quantitativas dos constituintes da biomassa através da interação das ondas eletromagnéticas do infravermelho próximo com a amostra. Este trabalho tem como objetivo fornecer uma revisão sobre a técnica da espectroscopia no infravermelho próximo e sua aplicação no setor florestal. A técnica está presente em praticamente todas as áreas, devido ao nível de desenvolvimento que esta tecnologia atingiu nos últimos anos. A espectroscopia NIR tem se mostrado uma ferramenta rápida e eficiente para substituição dos diversos ensaios que determinam a qualidade da madeira.
\end{abstract}

Palavras-chave: espectroscopia; infravermelho próximo; madeira.

\begin{abstract}
The knowledge of wood properties is the fundamental importance for the indication of the potential and use of this material. In the search for new alternatives for a fast, simple and reliable characterization, there are the non-destructive evaluations of wood. The near infrared spectroscopy (NIRS) has been used as a non-destructive method that allows qualitative and quantitative information of the constituents of biomass through the interaction of electromagnetic waves with near-infrared next to the sample. This work aims to provide a review of the technique of near infrared spectroscopy and its application in forestry. The technique is used in virtually all areas due to the level of development that this technology has reached in recent years. NIR spectroscopy has proved a quick and efficient replacement of several tests that determine the quality of the wood. This is a literature review and state of the art on the theme.
\end{abstract}

Keywords: spectroscopy; infrared; wood.

1. Engenheira Florestal, Dr., Professora Titular do Departamento de Engenharia e Tecnologia Florestal, Universidade Federal do Paraná, Campus III, Av. Pref. Lothário Meissner, 900, CEP 80210-170, Curitiba (PR). gbmunize@ufpr.br

2. Engenheiro químico, Dr., Pesquisador da EMBRAPA Florestas, Estrada da Ribeira, km 111, CEP 83411-000, Colombo (PR).wmagalha@cnpf.embrapa.br

3. Engenheira Industrial Madeireira, MSc., Professora Assistente do Departamento de Engenharia e Tecnologia Florestal, Universidade Federal do Paraná, Campus III, Av. Pref. Lothário Meissner, 900, CEP 80210-170, Curitiba (PR).mayaraelita@ufpr.br

4. Engenheira Florestal, MSc., Professora Colaboradora do Departamento de Engenharia Florestal, Universidade Estadual do Centro-Oeste, PR 153 Km 7 - Riozinho, CEP 84500-000, Irati (PR). licvianna@hotmail.com Recebido para publicação em 2/09/2009 e aceito em 10/04/2011 


\section{INTRODUÇÃO}

As propriedades tecnológicas da madeira e de seus derivados são determinadas através de uma série de ensaios que envolvem diferentes técnicas analíticas. Geralmente, estes ensaios são feitos por amostragem, usando métodos tradicionais que são, na sua maioria, onerosos, laboriosos e destrutivos, impedindo muitas vezes a utilização futura do material. Para as indústrias de base florestal isto dificulta a realização de um controle minucioso da qualidade de seus produtos.

Embora os resultados obtidos por estas técnicas sejam considerados precisos e confiáveis para indicação do potencial tecnológico da madeira de uma espécie ou clone, na maioria dos casos, as metodologias e técnicas empregadas impedem a análise de um grande número de amostras, inviabilizando também a caracterização desta matéria-prima em programas de melhoramento genético.

Como é de conhecimento amplo, a madeira é um material altamente heterogêneo, apresentando variações em suas propriedades entre espécies e dentro de uma espécie (KOLLMANN e CÔTE, 1968), entre clones e dentro de um mesmo clone (TOMAZELLO FILHO, 1985; EVANS et al., 2000) e até dentro de um mesmo indivíduo nos sentidos radial e longitudinal. Para abranger toda esta variabilidade e para obter resultados representativos, é necessário ensaios com um grande número de amostras, ou ainda, em alguns casos técnicas que permitam análise de todo o material.

Por este motivo o desenvolvimento de novas e sofisticadas técnicas instrumentais e metodologias de caracterização que possibilitem análises em tempo real (on-line) e determinação de várias propriedades simultaneamente, de maneira não destrutiva, vêm se tornando cada vez mais conhecidas e exploradas pelas indústrias, permitindo dessa forma adequada caracterização da madeira, de forma mais fácil, rápida e precisa. Nestes ensaios, conhecidos como não destrutivos, as informações sobre o material são obtidas por medidas indiretas, o que reduz o custo e o tempo despendido nas análises de rotina em laboratórios. Dentre os métodos não destrutivos, a espectroscopia, no modo de reflexão, destaca-se por ser técnica de obtenção rápida de resultados e possibilidade de análises on-line, ou em tempo real, demonstrando ser ferramenta adequada para a gestão e tomada de decisões no processo produtivo das indústrias.

\section{ESPECTROSCOPIA NO INFRAVERMELHO PRÓXIMO}

Frederick William Herschel, astrônomo e músico inglês, foi o responsável pela descoberta da região do infravermelho próximo, em 1800. Sabia-se que os espectros no infravermelho armazenavam uma gama de informações sobre a amostra e, portanto, apresentavam elevado potencial para serem empregados nos mais diversos tipos de análises químicas elou físicas (PASQUINI, 2003).

$\mathrm{Na}$ década de 30 foram registrados relatos da utilização desta técnica, porém, merece destaque, pela importância dos seus trabalhos, o grupo de pesquisadores de Karl Norris, que durante a década de 60 iniciaram suas pesquisas aplicando a espectroscopia no infravermelho próximo (NIRS) como novo método de determinação da umidade em produtos agrícolas (BOKOBZA, 1998). Após os trabalhos de Karl Norris, a técnica NIRS foi fortemente impulsionada nas décadas de 80 e 90 pelo avanço da instrumentação relacionada ao método e desenvolvimento dos microcomputadores, participando do nascimento da Quimiometria, ciência que utiliza em conjunto os métodos matemáticos, estatísticos e informáticos com a finalidade de obter informações quimicamente relevantes a partir dos dados químicos medidos, e representar e apresentar essa informação (WOLD e SJÖSTRÖM, 1998).

Atualmente, a espectroscopia no NIR tem sido frequentemente aplicada como método analítico que fornece resultados eficientes para a determinação de moléculas orgânicas e variáveis qualitativas. Esta técnica está presente em praticamente todas as áreas, devido ao nível de desenvolvimento que a tecnologia atingiu nos últimos anos. Para se ter uma ideia da ampla diversidade de aplicações da espectroscopia no NIR, os setores de maior utilização incluem as áreas agrícola, alimentícia, médica, têxtil, de cosméticos, de polímeros, de tintas, ambiental, petroquímica, farmacêutica e florestal.

O espectro da radiação eletromagnética abrange as ondas de rádio, as micro-ondas, o infravermelho, a luz visível, os raios ultravioleta, os raios $\mathrm{X}$ e os raios gama. O Infravermelho Próximo (NIR, do inglês, Near Infrared) é a denominação dada à região do espectro eletromagnético "mais 
próximo" à região visível, onde as vibrações moleculares que resultam em transições harmônicas (overtones) são responsáveis pela absorção nesta região (BURNS e CIURCZAK, 2008).

A técnica NIR é baseada na espectroscopia vibracional e consiste na exposição de várias amostras à radiação eletromagnética na região de comprimento de onda, que varia de 750 a $2.500 \mathrm{~nm}$, para se obter os espectros de absorbâncias. Pode ser feita, então, a correlação destes com os resultados de análises laboratoriais convencionais para a geração de um modelo estatístico de forma a explicar a maioria das informações contidas nos espectros (WILLIAMS e NORRIS, 2001). Por meio desta ferramenta são obtidas informações qualitativas e quantitativas dos constituintes químicos da biomassa decorrente da interação da onda eletromagnética do infravermelho próximo com a amostra (PASQUINI, 2003). Para a caracterização de uma amostra desconhecida, basta a obtenção de seu espectro e o uso do modelo estatístico previamente construído para a predição ou classificação de suas propriedades.

De forma geral, as ocorrências espectrais na região NIR provêm de ligações das moléculas em que participam o hidrogênio, o que torna a técnica útil para a determinação de compostos orgânicos contendo principalmente ligações $\mathrm{C}-\mathrm{H}, \mathrm{N}-\mathrm{H}, \mathrm{S}-\mathrm{H}$ e O-H (SCAFI, 2005). A espectroscopia no NIR apresenta algumas desvantagens, dentre elas é possível citar que as determinações quantitativas somente são possíveis através de prévia correlação entre os valores do parâmetro de interesse para um determinado grupo de amostras e os espectros obtidos. A técnica é dependente de metodologias analíticas bem estabelecidas para a determinação desse parâmetro durante a etapa de calibração.

Por outro lado, o método mostra uma série de vantagens, pois é rápido (um minuto ou menos na leitura por amostra), não invasivo, adequado para uso em linha de produção, permitindo análises on-line ou em tempo real, exige preparo mínimo da amostra e possui instrumental simples, sendo possível de ser transportado a longas distâncias, visando seu uso em operações de fiscalização e verificação da autenticidade de amostras.

Os espectros no infravermelho próximo apresentam difíceis atribuições de picos e são frequentemente largos e sobrepostos, devidos às bandas de combinações e sobretons. Os dados espectrais são tratados, atualmente, com o uso de técnicas multivariadas (SCAFI, 2005).

\section{MÉTODOS DE ANÁLISE MULTIVARIADA}

Existem diversas técnicas de estatística multivariada, com as mais variadas aplicações. Tais métodos podem ser classificados em dois tipos principais: os métodos de análise exploratória e os métodos de calibração multivariada. A aplicação de um ou outro método, ou até mesmo a combinação dos dois, depende da natureza do problema que se deseja resolver, ou do tipo de informação que se pretende obter (MALINOWSKI, 1991).

Para que as técnicas multivariadas possam ser aplicadas é necessário que os dados do sistema analisado estejam organizados em matrizes. A matriz de espectros original X, formada por $\mathbf{m}$ linhas (amostras) $\mathbf{n}$ colunas (variáveis), é decomposta em duas matrizes de variações: a matriz de loadings (pesos) e a de scores (escores). A matriz de scores é constituída de $\mathbf{m}$ linhas e $\mathbf{d}$ colunas, onde d corresponde ao número de componentes principais. A matriz de loadings é constituída de d linhas e $\mathbf{n}$ colunas. Os espectros originais podem ser considerados como combinações lineares das variações dos espectros (pesos) onde os escores representam a contribuição de cada espectro original (BURNS e CIURCZAK, 2008).

\section{Análise Exploratória}

Ao se deparar com um conjunto de dados, principalmente aquele muito extenso, é necessária análise prévia inicial para avaliar a qualidade das informações disponíveis.

Os métodos de análise exploratória têm por objetivo realizar a avaliação inicial dos dados para descobrir que tipo de informação pode-se extrair deles e, assim, definir as diretrizes para um tratamento mais aprofundado. Isto é feito através da utilização de algoritmos que permitem reduzir a dimensão dos dados, ou organizá-los numa estrutura que facilite a visualização do conjunto, de forma global. Para realizar esta análise inicial, os dois métodos estatísticos mais conhecidos são a Análise por Componentes Principais (PCA) e a Análise Hierárquica de Agrupamentos (HCA) (MALINOWSKI, 1991).

\section{Análise por Componentes Principais (PCA)}

A análise por componentes principais (PCA) é um dos métodos mais comumente empregados na análise de informações (FERREIRA, 2002).

Quando se aplica um algoritmo de PCA num conjunto de variáveis, como por exemplo, 
espectros no infravermelho, o conjunto original destas variáveis é substituído por um novo conjunto de variáveis denominado de Componentes Principais (CPs). A principal característica deste novo conjunto é a ortogonalidade, porém, o mesmo é facilmente reconstruído a partir da combinação linear das variáveis originais (espectros). Como vantagem, o novo conjunto de variáveis (CPs) geralmente concentra a maior parte da informação (variância) em poucas variáveis, diminuindo assim a dimensionalidade dos dados, sem perda significativa da informação química. As componentes principais podem fornecer a percentagem de variância contida em cada fator. Todas as componentes juntas em um modelo fornecem 100 \% de variância e a maior percentagem é explicada pela primeira componente principal.

A utilização desta análise vem se mostrando eficaz na discriminação de espécies de um mesmo gênero, as quais muitas vezes apresentam semelhanças em suas características anatômicas, sendo difícil a identificação no nível de espécie sem o seu material botânico. A Figura 1 ilustra o gráfico de scores de uma análise por PCA de amostras do gênero Dalbergia, onde seis espécies foram discriminadas. Para a construção deste modelo foram coletados espectros NIR, em amostras de madeira maciça, pertencentes ao acervo da xiloteca da Universidade Federal do Paraná, provenientes de diferentes regiões brasileiras. Esta análise prévia foi realizada pelos autores deste artigo de revisão.

\section{Calibração Multivariada}

A escolha de qual método de calibração utilizar está relacionada com o conjunto de dados investigados. Os mais comuns são Regressão Linear Múltipla (Multiple Linear Regression - MLR), Regressão de Componentes Principais (Principal Component Regression - PCR) e Regressão por Projeção de Variáveis Latentes (Projection to Latent Structure - PLS). Em todos estes dados, observase relação linear entre as variáveis espectrais e a propriedade a ser determinada .. (se estiver relacionado aos métodos) (PASQUINI, 2003).

\section{Projeção de Variáveis Latentes (PLS - Projection to Latent Structure)}

O método PLS é um método de regressão que fornece modelos que relacionam os blocos de variáveis X e Y. Deste modo, as informações das medidas espectrais e das concentrações ou propriedades são utilizadas simultaneamente na fase da calibração. É baseado no método PCA e utiliza as componentes principais para modelar os diferentes analitos e interferências nos dados de X.

A regressão através do método PLS pode solucionar problemas de colinearidade com satisfatória habilidade de previsão. Por este motivo esta técnica é adequadamente aplicada em dados espectrais. As informações das variáveis são comprimidas e, como consequência, os modelos são mais fáceis de serem interpretados e os ruídos espectrais podem ser mantidos fora do modelo, na

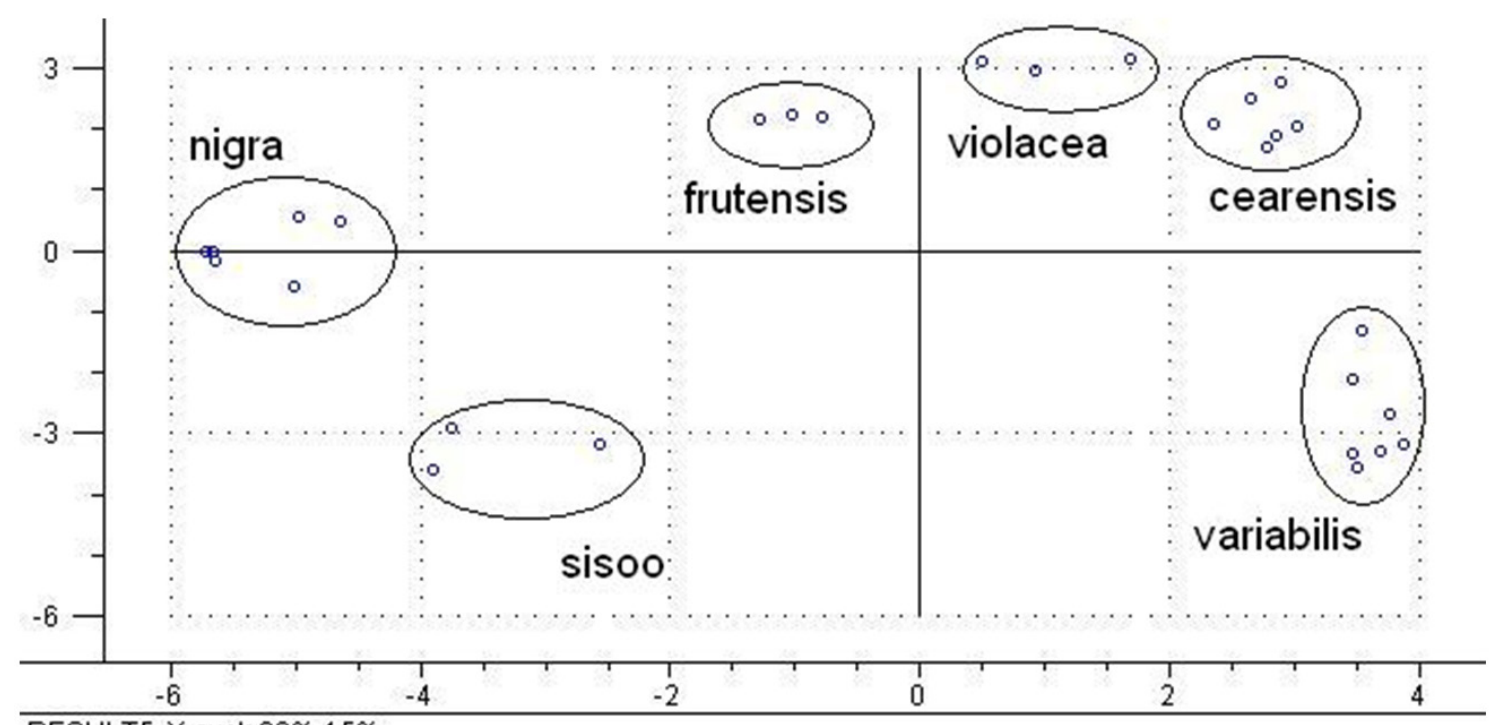

RESULT5, X-expl: 33\%,15\%

FIGURA 1: Análise por Componentes Principais (PCA) para discriminação de seis espécies do gênero Dalbergia.

FIGURE 1: Analysis of PCA for discrimination of six species of the genus Dalbergia. 
forma de resíduos (MARTENS e TORMOD, 1989).

A regressão PLS segue as etapas de calibração: a validação interna e a validação externa. Durante a calibração são utilizados os espectros de absorção das amostras do conjunto de calibração para a construção de um modelo matemático que melhor ajuste os dados espectrais aos valores de referência do parâmetro desejado. A validação interna avalia o erro de previsão de amostras que pertencem ao conjunto de calibração, também conhecida como validação cruzada. Finalmente, a validação externa avalia o poder de previsão do modelo construído utilizando amostras externas ao conjunto ou que não participaram da etapa de calibração. Esse último conjunto de amostras é conhecido também como conjunto de validação (SCAFI, 2005).

A etapa de validação é extremamente importante, pois os resultados dessa etapa indicarão se o modelo construído é adequado ou não para efetuar a determinação do parâmetro desejado em previsões futuras.

Para analisar a robustez dos modelos PLS desenvolvidos são avaliados os erros padrão na calibração (SEC), na validação cruzada (SECV) e na predição (SEP). O SEC (Standard Error of Calibration), calculado a partir das amostras de calibração é uma medida do erro na modelagem. O erro padrão na validação cruzada SECV (Standard Error of Cross Validation) é obtido a partir das amostras da validação cruzada e o SEP (Standard Error Prediction), é o erro padrão calculado a partir das amostras do conjunto de predição (NIEMEYER et al., 1992).

Outros parâmetros considerados para a seleção de modelos multivariados são o coeficiente de correlação $(\mathrm{R})$ e a relação de desempenho do desvio (RPD), obtida pela relação entre o desvio dos dados obtidos por análise convencional (Sdev) e o erro padrão da validação externa (SEP). O RPD fornece padronização do erro padrão da previsão e torna possível a comparação de diferentes propriedades (WILLIAMS e SOBERING, 1993).

A robustez do método PLS é uma característica importante, já que permite que novas amostras sejam incluídas ou retiradas do modelo de calibração sem alteração significativa do mesmo (GELADI e KOWALSKI, 1986). Isso é ideal para processos industriais onde é comum, devido a vários fatores, ocorrerem variações nas propriedades.

Em 1995, a American Standard for Testing and Materials (ASTM) lançou a norma ASTM 1655-05, chamada de Práticas Padrão para Análises
Quantitativas Multivariadas por Infravermelho (Médio e Próximo) que guia o processo de calibração multivariada.

\section{PESQUISAS DO SETOR FLORESTAL}

O uso da espectroscopia no infravermelho próximo (NIRS) tornou-se um método rápido para previsão e classificação das propriedades da madeira (SCHIMLECK et al., 2007). Nesse setor, os primeiros trabalhos utilizando a espectroscopia no infravermelho próximo e técnicas de análise multivariada foram voltados para predição da composição química da madeira e propriedades do papel. Entretanto, estudos comprovam também o sucesso de aplicação deste método na determinação das propriedades anatômicas, físicas, e mecânicas da madeira.

Birkett e Gambino (1989), na África do Sul, calibraram modelos para estimar o número Kappa de diferentes tipos de polpas de processos Kraft e encontraram coeficientes de correlação variando de 0,89 a 0,93 . Wright et al. (1990) trabalhando com polpas de 14 espécies de Pinus calibraram modelos para previsão do rendimento e o conteúdo de celulose com valores de coeficientes de determinação de 0,66 e 0,73 respectivamente. Raymond et al. (2001) determinaram o rendimento da polpa celulósica. Os autores desenvolveram modelos usando amostras de diferentes alturas e diferentes sítios para as espécies de Eucalyptus globulus e Eucalyptus nitens, encontrando valores de coeficientes de determinação entre 0,12 a 0,93 .

Schultz e Burns (1990) compararam os equipamentos FTIR e o NIR para calibrar modelos de previsão das propriedades químicas da madeira. Através de serragens de Pinus taeda e Liquidambar styraciflua mostraram que o NIR foi a técnica mais rápida e apresentou valores de coeficientes de correlação superiores para a predição do teor de celulose, lignina e hemicelulose.

Sefara et al. (2000) desenvolveram modelos para predição do rendimento da polpa celulósica para diferentes clones de Eucalyptus grandis da África do Sul. O melhor coeficiente de correlação foi de 0,89 para os espectros coletados em madeira sólida (cunhas).

Kihara et al. (2002) e Takayama et al. (1997) caracterizaram a lignina em madeira usando FTR-NIR. Jones et al. (2006) conseguiram predizer os teores de celulose, glucanas, xiloses, manoses, e lignina usando a espectroscopia na região do NIR. 
Terdwongworakul et al. (2005) Yeh et al. (2005) usaram a técnica quimiométrica para predizer propriedades químicas da madeira, recomendando o seu emprego para determinação do rendimento de polpa, teores de $\alpha$-celulose, pentosanas e lignina.

A espectroscopia na região do NIR pode predizer o grau de cristalinidade da celulose na madeira. Tsuchikawa e Siesler (2003a e b) usaram o FT-NIR para correlacionar espectros na região do NIR com a cristalinidade de celulose em madeira de folhosas e também de coníferas. Nesse estudo mostraram que as propriedades mecânicas da madeira são ditadas pelas interações entre os seus principais componentes químicos, celulose, lignina e hemicelulose.

No Brasil, as pesquisas com estas técnicas também estão diversificadas, Nisgoski (2005) avaliou o uso do NIR no estudo de características da madeira e do papel de clones de Pinus taeda L. As melhores correlações na calibração foram obtidas para massa específica básica $(0,87)$ e para o comprimento dos traqueoides axiais $(0,73)$.

Samistraro (2008) com o uso da espectroscopia no infravermelho próximo (NIR) e técnicas de análise multivariada obteve correlações entre 0,64 a 0,97 para a predição das propriedades físicas do papel Kraft de escala industrial. Hein (2008) avaliou diferentes tipos de amostragens, desde discos até serragem com diferentes granulometrias, de Eucalyptus urophylla para previsão de propriedades químicas e físicas, ajustando modelos com coeficientes de determinação entre 0,65 e 0,88 .

Rials et al. (2002) usaram a técnica para a predição das propriedades mecânicas de painéis MDF (Medium Density Fiberboard), encontrando altos índices de correlação para o MOE (Módulo de Elasticidade) e MOR (Módulo de Ruptura) destes painéis, 0,80 e 0,82 , respectivamente.

Thumm e Meder (2001) com amostras de Pinus radiata e espectros obtidos na face tangencial na região de 400 a $2500 \mathrm{~nm}$, desenvolveram modelos de calibração para predição da propriedade mecânica de compressão e obtiveram coeficiente de correlação de 0,74 para os espectros pré-tratados com $1^{\text {a }}$ derivada. Com espectros obtidos na madeira sólida de Pinus, Kelley et al. (2004a) desenvolveram modelos para predição das propriedades químicas e mecânicas. Ao trabalharem com o espectro todo, na faixa de 500 a $2400 \mathrm{~nm}$, os coeficientes de correlação para o MOE e MOR foram de 0,85 e 0,77 , respectivamente. Posterior seleção espectral foi definida na faixa de 650 a $1150 \mathrm{~nm}$ e os novos modelos de calibração apresentaram resultados bem similares aos já calibrados.

Kelley et al. (2004b) desenvolveram modelos para previsão do MOE e MOR de seis espécies: Pinus taeda, Pinus palustris, Pinus elliottii, Pinus echinata, Pinus ponderosa e Pseudotsuga menziesii e mostraram que, por meio de um único modelo, é possível prever estas propriedades das diferentes espécies. Para o modelo desenvolvido com quatro das seis espécies, obtiveram $\mathrm{R}^{2}$ de 0,87 e 0,88 para MOE e MOR, respectivamente.

Via (2004) realizou vasta pesquisa com a espectroscopia no infravermelho próximo, desenvolvendo modelos para predição das propriedades físicas, mecânicas e anatômicas com amostras de Pinus palustris, onde avaliou diferentes técnicas de regressão e a influência de amostras contaminadas por fungos manchadores nos modelos. Os valores de $\mathrm{R}^{2}$ variam de 0,47 e 0,88 e a inserção de amostras por fungos não interferiu na predição das propriedades.

A espectroscopia na região do NIR pode predizer propriedades anatômicas como comprimento de fibra e ângulo microfibrilar. Schimleck e Evans $(2002,2004)$ e Schimleck et al. $(2003,2005)$ estimaram o ângulo microfibrilar, rigidez, densidade aparente e características morfológicas em rolos de incremento de Pinus através da espectroscopia na região do NIR, usando como valor de referência medidas realizadas com a metodologia SilvScan desenvolvida no Commonwealth Scientific and Industrial Research Organisation (CSIRO) da Austrália.

Jones et al. (2005) estimaram, em amostras de Pinus taeda, o ângulo microfibrilar, a massa específica básica e a resistência à compressão. Foram desenvolvidos modelos com diversos pré-tratamentos e os coeficientes de correlação encontrados variaram de 0,79 a 0,93 .

O uso de espectroscopia para a predição do comprimento de fibras tem recebido pouca atenção dos programas de melhoramento florestais devido à escassez de estudos. Alguns trabalhos mostram que o uso de certas técnicas para tratamento prévio dos espectros, como a correção ortogonal do sinal (OSCOrthogonal Signal Correction), pode melhorar o poder de predição das técnicas quimiométricas aplicadas em propriedades anatômicas e físicas de madeira, do papel e da polpa (WOLD et al., 1998; MARKLUND e HAUKSSON, 1999; HAUKSSON et al., 2001).

Adespeito de quase a totalidade dos trabalhos 
usarem a reflectância difusa, principalmente por esta não exigir pré-tratamento das amostras, Sykes et al. (2005) mostraram que é possível obter curvas de calibração em madeira por transmitância. As amostras eram lâminas de madeira obtidas em micrótomo com cerca de $200 \mu \mathrm{m}$ de espessura. Estes autores avaliaram o uso do NIR para predição do teor de $\alpha$-celulose, comprimento de fibra, coarseness, e teor de lignina em madeira de Pinus taeda. Para a propriedade comprimento de fibra, os autores encontraram um coeficiente de determinação para a curva de calibração de 0,43 e um erro padrão de predição, usando amostras externas àquelas usadas para a calibração de $0,491 \mathrm{~mm}$, sendo que o valor do comprimento médio das fibras era de 1,598 $\mathrm{mm}$. Valores que podem ser considerados satisfatórios, quando se leva em consideração a imensa variabilidade natural do material, as dificuldades laboratoriais e somadas aos acúmulos de erros em todo o sistema para a mensuração de elemento anatômico.

Schimleck et al. (2004) encontraram valores para os coeficientes de determinação de 0,88 e 0,96 , para as curvas de calibração para as médias aritmética e, ponderada do comprimento de fibra, respectivamente. Os espectros foram obtidos de amostras na forma de rolos de incremento. Todavia, para a predição externa, as curvas de calibração apresentaram coeficientes de determinação de 0,58 e 0,86 , com erro padrão de predição de 0,17 e $0,25 \mathrm{~mm}$, respectivamente.

Via et al. (2005) obtiveram curva de calibração satisfatória, usando espectros de absorbância na região do NIR para amostras de lenho juvenil de madeira de Pinus palustris. Segundo esses autores, a falha no modelo de predição para amostras do lenho adulto está no fato do espectro na região do NIR trazer alguma informação química da lignina e da celulose que estão correlacionadas com o comprimento de fibra. Ainda assim, os coeficientes de determinação para as curvas de calibração e de validação externa foram de 0,76 e 0,65 , respectivamente.

Marklund e Hauksson (1999) usaram pó de madeira para obtenção de espectros na região do NIR e correlacionaram com comprimento de fibra. Os coeficientes de determinação para as curvas de calibração e predição externa foram de 0,775 e 0,727 , respectivamente, após o uso de correção ortogonal do espectro. Hauksson et al. (2001) também usando a mesma técnica anterior em madeira de Picea abies alcançaram coeficientes de determinação para a predição externa das médias ponderadas dos comprimentos de fibras de 0,722 e 0,840 , com erros de predição externa de 2,13 e $0,63 \mathrm{~mm}$, respectivamente.

A espectroscopia na região do NIR é uma técnica não destrutiva de análise que tem sido usada com sucesso para predizer propriedades mecânicas de madeira. Kelley et al. (2004a) usou a técnica para medir as propriedades químicas e mecânicas de Pinus taeda. Os coeficientes de correlação encontrados foram de 0,88 para o MOE, tanto usando faixas espectrais entre 500 e $2400 \mathrm{~nm}$, como entre 650 e $1150 \mathrm{~nm}$. Resultados similares foram encontrados por Schimleck et al. (2001a), Thumm e Meder (2001), Meder et al. (2002), Gindl et al. (2002), Hauksson et al. (2001) e Hoffmeyer e Pedersen (1995).

Kelley et al. (2004b) e Schimleck et al. (2001b) desenvolveram modelos similares para predizer propriedades de diferentes espécies de coníferas e folhosas, a partir de dados espectrais na faixa do NIR. Esses trabalhos mostraram que um modelo único pode ser construído a partir de diferentes espécies, desde que ele seja usado para espécie que faça parte do mesmo conjunto de espécies usadas na calibração. Schimleck et al. (2005) usaram a espectroscopia na região do NIR para predizer, de forma bastante robusta, correlação com ângulo microfibrilar e rigidez para madeiras de Pinus radiata e Pinus taeda. A correlação é boa mesmo quando não há uma grande variação da densidade, sendo a lignina, a celulose e talvez a hemicelulose os componentes da madeira responsáveis pela predição das propriedades.

André et al. (2006) mostraram que a espectroscopia na região do NIR pode ser usada para prever o estado de tensões em pequenas peças de madeira seca de yellow poplar (Liriodendron spp). Nesse estudo, obtiveram-se espectros na região do NIR em peças submetidas a diferentes cargas em ensaios de quatro pontos. Os espectros foram obtidos tanto na face tracionada, como na face comprimida. A técnica mostrou-se eficiente para distinguir entre madeira tracionada e comprimida. Além disso, permitiu predizer os estados de tensões com coeficientes de correlação maior que 0,98. Outro aspecto a ser considerado é que o experimento foi feito na faixa em que o estado de tensões está abaixo do limite de elasticidade da madeira.

Fujimoto et al. (2007) mostraram que a espectroscopia na faixa do NIR pode ser usada para predizer propriedades de rigidez e resistência 
mecânica da madeira de híbridos de Larix. Meglen e Kelley (2003) advogam a possibilidade de predição de propriedades mecânicas em madeira ainda verde. A tensão de crescimento medida por Bailleres et al. (2002) e a sua correlação com espectros de NIR são questionáveis. O próprio trabalho cita em suas discussões, que a tensão medida no perímetro externo da árvore (trata-se da tração) e ignora possíveis compressões que poderiam existir no interior do tronco. Além do mais, a amostra que teve o seu espectro coletado, na verdade, não corresponde a que designou à medida da tensão. Ainda assim, a correlação foi de 0,64 e apresentou um erro de predição igual ao erro da técnica convencional com RPD de 1,85. Schimleck et al. (2003) admitem um RPD muito menor para programas de melhoramento em seu screening inicial.

Tsuchikawa et al. (2005) estimaram usando FT-NIR e quimiometria a densidade e a resistência mecânica de madeira, tanto na faixa de deformação elástica, como na plástica, representados pelo $\mathrm{MOE}$ e pela máxima resistência à tração. A espectroscopia na região do NIR pode predizer propriedades químicas da madeira. Michell e Schimleck (1996) estabeleceram muitas correlações entre bandas na região do NIR e àquelas na região do infravermelho médio, confirmando que as bandas na região do NIR são combinações de várias bandas fundamentais. Além do mais, Schimleck et al. (1997) mostraram que a composição química de onze amostras de madeira de eucalipto estão correlacionadas com bandas nos espectros na região do NIR.

Abrahão (2005), com espectros de UV-Vis-NIR da madeira sólida de Eucalyptus urophylla combinada à ferramenta quimiométrica PCR, encontrou $\mathrm{R}^{2}$ 0,89 e RMSEP de $5 \%$ para a propriedade massa específica básica.

Barcellos (2007) trabalhou com NIR para determinação das propriedades do carvão vegetal. Sete espécies nativas e cinco do gênero Eucalyptus foram avaliadas, obtendo-se coeficientes de correlação iguais a 0,$97 ; 0,96$ e 0,91 para previsão de teores de carbono fixo, materiais voláteis e poder calorífico, respectivamente.

Muitas das pesquisas desenvolvidas já foram registradas como patentes. Engström e Hedqvist (1999) patentearam a utilização de técnicas espectroscópicas combinadas à calibração multivariada para predição das propriedades da madeira, vislumbrando sua utilização para monitorar processos industriais de painéis.

Meglen e Kelley (2003) registraram que, por meio da espectroscopia VIS/NIR, é possível desenvolver modelos para predição das propriedades mecânicas, tanto para amostras de madeira como quanto para árvores ainda no plantio.

Cowan et al. (2007) desenvolveram metodologia baseada na técnica de espectroscopia no infravermelho para monitorar a condição da colagem de painéis estruturais de madeira.

Como visto, a utilização da espectroscopia na análise da madeira e seus subprodutos aumentou acentuadamente nos últimos anos, juntamente com a possibilidade de utilizá-la diretamente no processo. Skoglund et al. (2004) e Nenrisksen et al. (2005) avaliaram o uso do NIR para controle on-line de medidas de propriedades de madeira na entrada de um digestor contínuo em indústria de celulose. Caldeira et al. (2006) desenvolveram sistema por espectroscopia no infravermelho para controle online de teor de umidade e massa específica para cavacos.

\section{CONSIDERAÇÕES FINAIS}

A técnica NIRS vem se mostrando eficiente para substituição dos diversos ensaios que determinam a qualidade da madeira, e o sucesso desta proposta esta relacionado fortemente à etapa de calibração, que dependerá da disponibilidade de padrões certificados e de amostras de referência. Além disso, um grande conjunto de amostras reais analisadas por metodologia padrão é de extrema importância para a validação dos métodos propostos.

As pesquisas para predição das propriedades da madeira e dos produtos de madeira com a espectroscopia no infravermelho vêm apresentando altas correlações e observando que em diversos seguimentos industriais a tecnologia NIR tornouse uma ferramenta indispensável para assegurar a qualidade de seus produtos è neste cenário que se acredita que as indústrias de base florestal devem seguir. Possivelmente com investimentos em futuras instalações de equipamentos de NIR para monitoramento on-line, onde será possível o controle, em altíssima velocidade, da qualidade dos produtos que chegam e saem das fábricas e, sobretudo, de lucratividade.

\section{REFERÊNCIAS BIBLIOGRÁFICAS} ABRAHÃO, C. P. Estimativa de
algumas propriedades da Madeira de Eucalyptus
urophylla por espectrometria. 2005.182 f. Tese 
(Doutorado em Ciências Florestais) - Universidade Federal de Viçosa, Viçosa, 2005.

ANDRÉ, N. et al. Assessment of wood load condition by near infrared (NIR) spectroscopy. Journal Material Science, v. 41, p. 1876-1886, 2006.

BAILLÈRES, H.; DAVRIEUX, F.; HAMPICHAVANT, F. Near infrared analysis as a tool for rapid screening of some major wood characteristics in a eucalyptus breeding program. Forestry Science, v. 59, p. 479-490, 2002.

BARCELlOS, D. C. Caracterização do Carvão Vegetal através do uso de Espectroscopia no Infravermelho Próximo. 2007. 140 f. Tese (Doutorado em Ciências Florestais) - Universidade Federal de Viçosa, Viçosa, 2007.

BIRKETT, J. A.; GAMBINO, M. J. T. Estimation of Pulp kappa number with near-infrared spectroscopy. Tappi Journal, v. 72, n. 9, p. 193-197, 1989.

BOKOBZA, L. Near Infrared Spectroscopy. Journal of Near Infrared Spectroscopy, v. 6, n. 1, p.3-17, 1998.

BURNS, D. A.; CIURCZAK, E. W. Handbook of near-infrared analysis. 3th ed. Boca Raton: CRC, 2008. 808 p.

CALDEIRA, A. F. et al. NIR on-line: an innovation in the VCP wood yard. Disponível em: <http:// www.celuloseonline.com.br/imagembank/Docs/ DocBank/Eventos/430/3CaldeiraOral.pdf.> Acesso em 12/04/2006.

COWAN, J. J.; LANDERS, A. G. Method using NIR spectroscopy to monitor components of engineered wood products. United States Patent, 7,279,684 B2, Oct.9, 2007.

ENGSTRÖM, B.; HEDQVIST, M. Prediction of the properties of board by using a spectroscopy method combined with multivariate calibration. United States Patent, 5,965,888, Oct. 12, 1999.

EVANS, J. L. W.; SENFT, J. F.; GREEN, D. W. Juvenile wood effect in red alder: analysis of physical and mechanical data to delineate juvenile and mature wood zones. Forest Products Journal. v. 50, n. $7 / 8$, p. $75-87,2000$.

FERREIRA, M. C. et al. Quimiometria I: Calibração Multivariada, um Tutorial, Química Nova, v. 22, p.724- 731, 1999.

FERREIRA, M. C. Multivariate QSAR. Journal Brazilian Chemical Society, São Paulo, v. 13, n. 6, p. 742-753, 2002.

FUJIMOTO, T.; YAMAMOTO, H.; TSUCHIKAWA, S. Estimation of wood stiffness and strength properties of hybrid larch by near infrared spectroscopy. Applied spectroscopy, v. 61, p. 882-888, 2007.

GELADI, P.; KOWALSKI, B. R. Partial LeastSquare regression: a tutorial. Analytica Chimica Acta, Amsterdam, v. 185, p. 1-17, 1986.

GINDL, W. et al. The relationship between Near Infrared Spectra of radial wood surfaces and wood mechanical properties. Journal Near Infrared Spectroscopy, v. 9, n. 4, p. 255, 2001.

HAUKSSON, J. B. et al. Prediction of basic wood properties for Norway spruce. Interpretation of Near Infrared Spectroscopy data using partial least squares regression. Wood Science and Technology, v. 35, n. 6, p. 475, 2001.

HEIN, P. R. G. Avaliação das Propriedades da Madeira de Eucalyptus urophylla por meio da espectroscopia no Infravermelho Próximo. 2008. 74 f. Dissertação (Mestrado em Ciências Florestais) -Universidade Federal de Lavras, Lavras, 2008.

HOFFMEYER, P.; PEDERSEN, J. Evaluation of density and strength of Norway spruce wood by near infrared reflectance spectroscopy. Holz als Roh- und Werkstoff, v. 53, p. 165, 1995.

JONES, P. D. et al. Nondestructive estimation of Pinus taeda L. wood properties for samples from a wide range of sites in Georgia. Canadian Journal of Forest Research, v. 35, p. 85-92, 2005.

JONES, P. D. et al. Nondestructive estimation of wood chemical composition of sections of radial wood strips by diffuse reflectance near infrared spectroscopy. Wood Science and Technology, v. 40, p. 709-720, 2006.

KELLEY, S. S. et al. Use of near infrared spectroscopy to predict the mechanical properties of six softwoods. Holzforschung, v. 58, n. 3, p. 252260, 2004b.

KELLEY, S. S. etal.Use of nearinfrared spectroscopy to measure the chemical and mechanical properties of solid wood. Wood Science and Technology, v. 38, p. 257-276, 2004a.

KIHARA, M. et al. Determination of the carbonyl groups in native lignin utilizing Fourier transform Raman spectroscopy. Spectrochimica Acta Part A: Molecular and Biomolecular Spectroscopy, v. 58, n. 10, p. 2213-2221, 2002.

KOLlMANN, F. R.; COTÉ, W. A. Principles of Wood science and technology. Berlin: SpringerVerlag, 1968, $592 \mathrm{p}$.

MALINOWSKI, E. R. Factor Analysis in Chemistry, 2th. ed. New York: John Wiley \& Sons, Inc., 1991.

MARKLUND, A.; HAUKSSON, J. B. Multivariate 
data analysis based on orthogonal signal correction and near infrared spectroscopy. Nordic Pulp and Paper research Journal, v. 14, n. 2, p. 140-148, 1999.

MARTENS H.; TORMOD N. Multivariate Calibration, New York: John Wiley \& Sons, 1989. MEDER R.; THUMM, A.; BIER, H. Veneer stiffness predicted by NIR spectroscopy calibrated using mini-LVL test panels. Holz als Roh- und Werkstoff, v. 60, n. 3, p. 159, 2002.

MEGLEN, R. R.; KELEY, S. S. Use of region of the visible and Near Infrared Spectrum to predict mechanical properties of wet wood and standing trees. United States Patent, 6,525,319 B2, Feb,25, 2003.

MICHELL, A. J.; SCHIMLECK, L. R. NIR spectroscopy of woods from Eucalyptus globulus. Appita Journal, v. 49, p. 23-26, 1996.

NENRISKSEN, H. C. et al. Simultaneous modeling o process variables and raw material properties as measured by NIR. A case study from cellulose production. Chemometrics and Intelligent Laboratory System, v. 77, p. 238-246, 2005.

NIEMEYER, J.; CHEN, Y.; BOLLAG, J. M. Characterization of humic acids, composts and peat by diffuse reflectance Fourier-transform infrared spectroscopy. Soil Science Society of America Journal, v. 56, p. 130-135, 1992.

NIGOSKI, S. Espectroscopia no infravermelho próximo no estudo de características da madeira e papel de Pinus taeda L. 2005. 160 f. Tese (Doutorado em Engenharia Florestal) - Setor de Ciências Agrárias, Universidade Federal do Paraná, Curitiba, 2005.

PASQUINI, C. Near infrared spectroscopy: fundamentals, practical aspects and analytical applications. Journal of the Brazilian Chemical Society, v. 14, n. 2, p. 198-219, 2003.

RAYMOND, C. A. et al. Nondestructive sampling of Eucalyptus globus and E. nitens for wood properties. III. Predict pulp yield using near infrared reflectance analysis. Wood Science and Technology, v. 35, p. 203-215, 2001.

RIALS, T, G.; KELLEY S, S.; SO C. L. Use of advanced spectroscopy techniques for predicting the mechanicals properties of wood composites. Wood and Fiber Science, v. 34, n. 3, p. 398-407, Jul 2002.

SAMISTRARO, G. Previsão das propriedades químicasdapolpa epapelKraftpor espectroscopia no Infravermelho Próximo (NIR) e regressão de mínimos quadrados parciais (PLS). 2008. $115 \mathrm{f}$. Dissertação (Mestrado em Engenharia Florestal) Setor de Ciências Agrárias,. Universidade Federal do Paraná. Curitiba, 2008.

SCAFI, S. H. F. Sistema de Monitoramento em Tempo Real de Destilações de Petróleo e Derivados Empregando a Espectroscopia no Infravermelho Próximo. 2005. 196 f. Tese (Doutorado em Química) - Instituto de Química, Universidade Estadual de Campinas, Campinas, 2005.

SCHIMLECK, L. R. et al. Estimation of microfibril angle and stiffness by near infrared spectroscopy using sample sets having limited wood density variation. IAWA Journal, v. 26, n. 2, p. 175-187, 2005.

SCHIMLECK, L. R. et al. Microfibril angle prediction of Pinus taeda wood samples based on tangencial face NIR spectra. IAWA Journal, v. 28, n. 1, p. 1-12, 2007.

SCHIMLECK, L. R. et al. Near-infrared spectra and chemical compositions of Eucalyptus globulus and Eucalyptus nitens plantation woods. Appita Journal, v. 50, p. 40-46, 1997.

SCHIMLECK, L. R. et al. Nondestructive estimation of tracheid length from sections of radial wood strips by near infrared spectroscopy. Holzforschung, v. 58, p. 375-381, 2004

SCHIMLECK, L. R.; EVANS, R. Estimation of microfibril angle of increment cores by near infrared spectroscopy. IAWA Journal, v. 23, n. 3, p. 225 234, 2002.

SCHIMLECK, L. R.; EVANS, R.; ILIC, J. Application of near infrared spectroscopy to a diverse range of species demonstrating wide density and stiffness variation. IAWA Journal, v. 22, n. 4, p. 415 - 429, 2001b.

SCHIMLECK, L. R.; EVANS, R.; ILIC, J. Estimation of Eucalyptus delegatensis wood properties by near infrared spectroscopy. Canadian Journal Forestry Resource, v. 31, n. 10, p. 1671-1675, $2001 \mathrm{a}$.

SCHIMLECK, L. R.; MORA, C.; DANIELS, R. F. Estimation of tracheid morphological characteristics of green Pinus taeda L. radial strips by near infrared spectroscopy. Wood and Fiber Science, v. 36, n. 4, p. 527-535, 2003.

SCHIMLECK, L.R.; EVANS, R. Estimation of Pinus radiata D. Don tracheid morphological characteristics by near infrared spectroscopy. Holsforschung, v. 58, p. 66-73, 2004.

SCHULTZ T. P.; BURNS D.A. Rapid secondary analysis of lignocellulose: comparison of near 
infrared (NIR) and Fourier transformation infrared (FTIR), Tappi journal, v. 73, n. 5, p.209 - 212, 1990.

SEFARA, N. L., CONRADIE, D.; TURNER, P. Progress in the use of near infrared absorption spectroscopy as a tool for the rapid determination of pulp yield in plantation eucalypts. Tappsa Journal, p. 15-17, Nov. 2000.

SKOGLUND, A. et al. On-line spectroscopy measurements of wood chips before a continuous digester. Chemometrics and Intelligent Laboratory system, v.70, p. 129-135, 2004.

SYKES, R. et al. Prediction of loblolly pine wood properties using transmittance near-infrared spectroscopy. Canadian Journal of Forest. Research, v. 5, p. 2423-2431, 2005.

TAKAYAMA, M. et al. Fourier transform Raman assigment of gauaiacyl and syringyl marker bands for lignin determination. Spectrochimica Acta Part A: Molecular and Biomolecular Spectroscopy, v. 53, n. 10, p. 1621-1628, 1997.

TERDWONGWORAKUL, A. et al. Rapid assessment of wood chemical properties and pulp yield of Eucalyptus camaldulensis in Thailand tree plantations by near infrared spectroscopy for improving wood selection for high quality pulp. Journal Wood Science, v. 51, p. 167-171, 2005.

THUMM, A.; MEDER, R. Stiffness prediction of radiata pine clearwood test pieces using near infrared spectroscopy. Journal of Near Infrared Spectroscopy, v. 9, n. 2, p. 117-122, 2001.

TOMAZELLO FILHO, M. Variação radial da densidade básica e da estrutura anatômica da madeira do Eucalyptus saligna e Eucalyptus grandis. IPEF, n. 29, p. 37-45, 1985.

TSUCHIKAWA, S. et al. Near-infrared spectroscopy study of the physiscal and mechanical properties of wood with meso- and micro-scale anatomical observation. Applied Spectroscopy, v. 59, n. 1, p. 86-93, 2005.

TSUCHKAWA, S.; SIESLER, H. W. Near-infrared spectroscopy monitoring of the diffusion process of deuterium -labeled molecules in wood. Part II: hardwood. Applied Spectroscopy, v. 57, n. 6, p. 675-681, 2003a.

TSUCHKAWA, S.; SIESLER, H. W. Near-infrared spectroscopy monitoring of the diffusion process of deuterium -labeled molecules in wood. Part I: softwood. Applied Spectroscopy, v. 57, n. 6, p. 667-674, 2003 b.

VIA, B. K. et al. Tracheid length prediction in Pinus palustris by means of near infrared spectroscopy: the influence of age. Holz als Rohund Werkstoff, v. 63, p. 231-236, 2005.

VIA, B. K. Modeling longleaf pine (Pinus palustris mill) wood properties using near infrared spectroscopy. 2004. 141 f. Tese (Doctor of Philosophy) - Agriculture and Mechanical College, The School of Renewable Natural Resources, Louisiana State University, USA, 2004.

WILLIAMS, P. C.; SOBERING, D. C. Comparison of commercial near infrared transmittance and reflectance instruments for analysis of whole grains and seeds. Journal of Near Infrared Spectroscopy, Sussex, v. 1, n. 1, p. 25-33, 1993.

WILLIAMS, P.; NORRIS, K. Near-infrared technology. $2^{\text {nd }}$ ed. Saint Paul:American Association of Cereal Chemistry, 2001. 296 p.

WOLD, S. et al. Orthogonal signal correction of near-infrared spectra. Chemometrics and Intelligent Laboratory Systems, v. 44, p. 175-185, 1998.

WOLD, S.; SJÖSTRÖM, M., Chemometrics, present and future success, Chemometrics and Intelligent Laboratory Systems, v. 44, p. 3-14, 1998.

WRIGHT, J. A.; BIRKETT, M. D.; GAMBINO, M. J. T. Prediction of pulp yield and cellulose content from wood samples using near infrared spectroscopy. Tappi Journal. v. 73, n. 8, p. 164166, 1990.

YEH, T-F. et al. Rapid screening of wood chemical component variations using transmittance nearinfrared spectroscopy. Journal Agriculture Food Chemistry, v. 53, p. 3328-3332, 2005. 